\title{
Use of 1,064-nm 0-switched Neodymium:Yttrium-aluminum- garnet Laser Therapy Assisted with Diamond Particle Suspension and Gold Microparticle Application for Acne Vulgaris and Enlarged Facial Pores
}

\author{
Hee Ung Park ${ }^{1}$ \\ Hangrae $\mathrm{Cho}^{2}$ \\ Sang Ju Lee ${ }^{3}$ \\ Han Kyoung $\mathrm{Cho}^{1}$
}

${ }^{1}$ Department of Dermatology, Hanyang University Medical Center, Myongji Hospital, Goyang, Korea ${ }^{2}$ Ohkims Dermatology Clinic, Goyang, Korea ${ }^{3}$ Yonsei Star Skin \& Laser Clinic, Seoul, Korea
Received September 4, 2021

Accepted October 11, 2021

\footnotetext{
Correspondence

Han Kyoung Cho

Department of Dermatology, Hanyang University Medical Center, Myongji Hospital, 55 Hwasu-ro, 14 beon-gil, Deogyang-gu, Goyang 10475, Korea Tel.: +82-31-810-7050

Fax: +82-31-810-7057

E-mail: trpchkahanmail.net

(C) Korean Society for Laser Medicine and Surgery

(c) This is an open access article distributed under the terms of the Creative Commons Attribution NonCommercial License (http://creativecommons.org/ licenses/by-nc/4.0) which permits unrestricted noncommercial use, distribution, and reproduction in any medium, provided the original work is properly cited.
}

Acne vulgaris is a common inflammatory skin disease of the pilosebaceous unit. It appears as lesions consisting of comedones, papules, pustules, and nodules of varying shapes and severity. In general, the first-line treatment for acne vulgaris includes topical and oral medication. Recently, various physical modalities have also been investigated. The use of laser therapy is steadily increasing because of its fewer side effects, short procedure time, and rapid results. In particular, laser therapy assisted with carbon suspension application is effective for acne vulgaris but may sometimes result in discomfort due to odor and dust formation during the procedure. Herein, we report that acne vulgaris and enlarged facial pores can be safely and effectively treated with laser therapy assisted with diamond particle suspension and gold microparticle application, which can address the discomfort caused by the carbon suspension application.

\section{Key words}

Acne vulgaris; Facial pore; Diamond particle suspension; Gold microparticle; Neodymium:yttrium-aluminum-garnet laser 


\section{INTRODUCTION}

Acne vulgaris is a common skin disease worldwide, and its sequelae can last for a lifetime. ${ }^{1-3}$ It remains a chronic problem for many patients, because it can cause psychological impairment. ${ }^{1,3}$ Topical and oral medications are considered the first-line treatment for acne vulgaris; however, they are limited in their effects and can have local or systemic side effects. ${ }^{2-4}$ Isotretinoin is one of the most common medications for acne treatment but cannot be used in pregnant women owing to its teratogenicity. ${ }^{1,34}$ Alternatively, light-based treatment is being studied steadily, and various laser therapies are being used to attempt to treat acne vulgaris. ${ }^{1,5,6}$ Laser therapy using microparticles has recently been reported to be effective in treating this skin condition. ${ }^{1-3,5-8}$

Herein, we report two cases showing significant treatment results of acne vulgaris with 1064-nm Q-switched neodymium:yttrium-aluminum-garnet (Nd:YAG) laser therapy assisted with diamond particle suspension and gold microparticle application.

\section{CASE REPORT}

\section{Case 1}

A 20-year-old woman presented with complaints of acne lesions, enlarged pores, and excessive sebum secretion on her face (Fig. 1A). She had no remarkable medical or family history.

After obtaining informed consent, we performed a total of three laser therapies on the entire face at 1-week intervals. Facial images were taken with a LED-sourced analyzer (Mark-Vu ${ }^{\circledR}$; PSI Plus Co., Ltd., Suwon, Korea) before and after laser therapy. Prior to the laser therapy, we applied a diamond particle suspension and gold microparticles (2nd derma Gold D ${ }^{\circledR}$, SimfleStick, Inc., Daegu, Koreal to the patient's face and then evenly spread them using a brush. The laser used was 1,064-nm Q-switched Nd:YAG laser (Spectra VRM IV; LUTRONIC Corp., Ltd., Goyang, Koreal. The parameters of the laser were as follows: $8-\mathrm{mm}$ spot size, $1.3-\mathrm{J} / \mathrm{cm}^{2}$ fluence, and $10-\mathrm{Hz}$ repetition rate. The total shot count was 3000 shots. After the laser therapy, the patient's sebum secretion, acne outbreaks, and size of the pores that had previously been markedly observed decreased, and the overall skin texture and redness of the face improved (Figs. 1B-D, Fig. 2).

\section{Case 2}

A 23-year-old woman presented with complaints of acne lesions and hyperpigmented scars on her forehead and cheeks that have been occurring since puberty (Fig. 3A). She had no remarkable medical or family history.

After obtaining informed consent, we performed the same treatment protocol as described above. However,
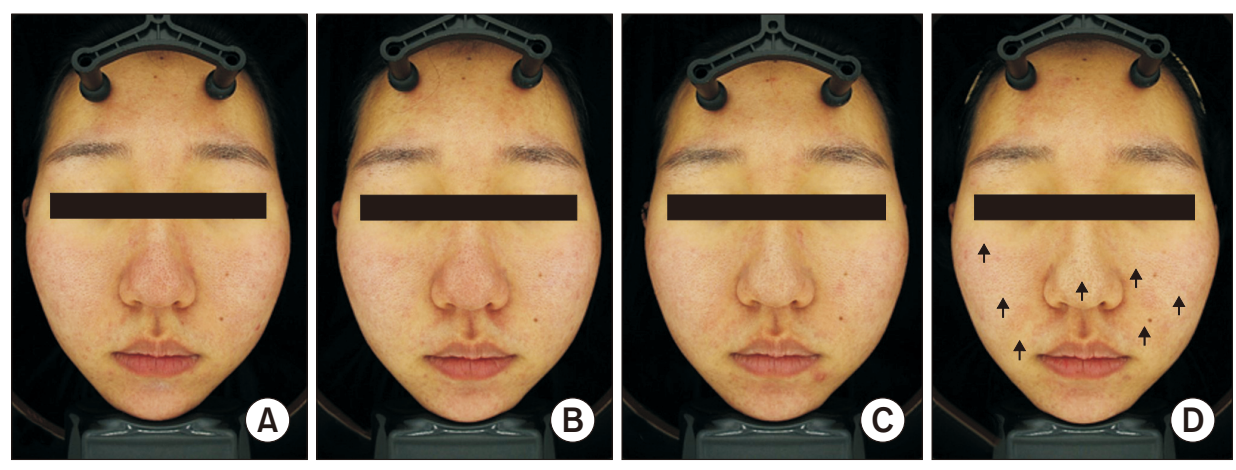

Fig. 1. A 20-year-old woman with enlarged facial pores and acne vulgaris: before the laser therapy (A), 7 days after the first laser therapy (B), 7 days after the second laser therapy (C), and 7 days after the third laser therapy, the redness and overall skin texture of the face improved (black arrow, D).
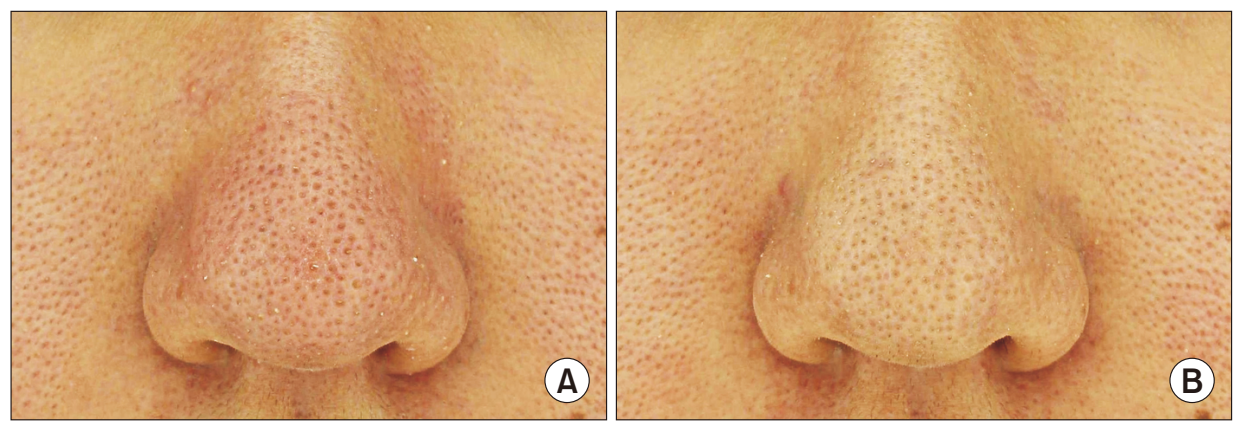

Fig. 2. A 20-year-old woman with enlarged facial pores and acne vulgaris: before the laser therapy (A), sebum secretion, acne outbreaks, and size of the pores decreased after the third laser therapy (B). 

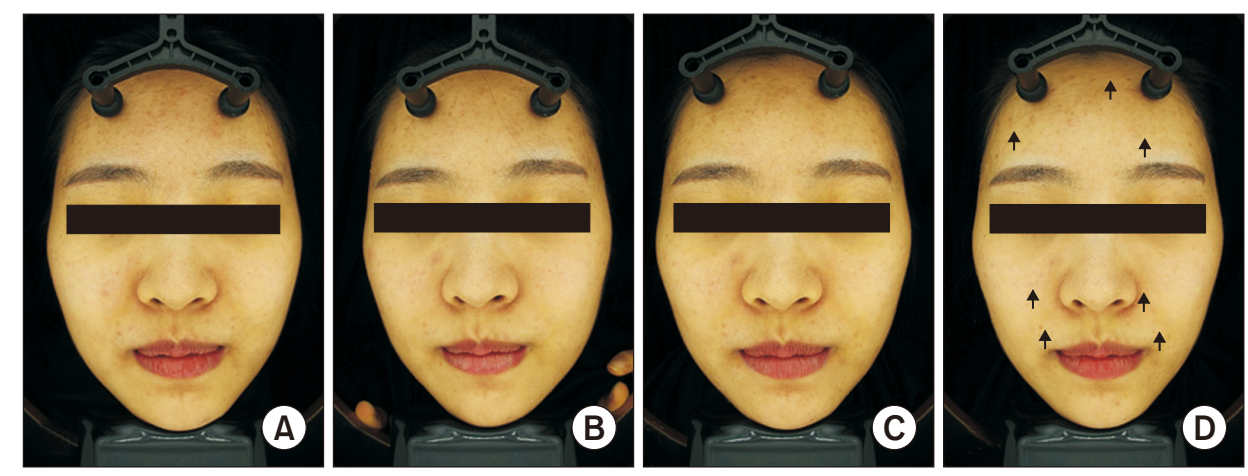

Fig. 3. A 23-year-old woman with hyperpigmented scars and acne vulgaris: before the laser therapy (A), 7 days after the first laser therapy (B), 7 days after the second laser therapy (C), and 7 days after the third laser therapy, the hyperpigmented scar and overall skin texture of the face improved (black arrow, D).
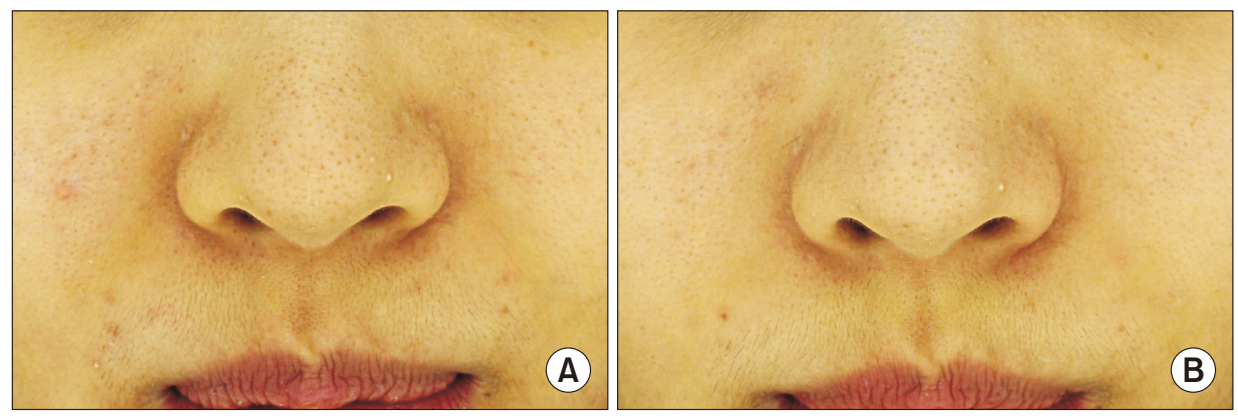

Fig. 4. A 23-year-old woman with hyperpigmented scars and acne vulgaris: before the laser therapy (A), hyperpigmented scars, acne outbreaks, and size of the pores decreased after the third laser treatment (B).

she was treated with a total of 3500 shots. After three laser therapies, the acne lesions decreased; the size of the pores shrunk; and the overall skin texture and hyperpigmentation scar of the acne lesion observed on the forehead improved visually (Figs. 3B-D, Fig. 4).

\section{DISCUSSION}

There are many different methods for treating acne vulgaris, including topical and oral medications and physical therapies. ${ }^{3}$ The light-based treatments for acne vulgaris include blue and red light therapy, intense pulsed light therapy, pulsed dye laser therapy, photodynamic therapy, and photothermal therapy. ${ }^{1-3,5-8}$ The mechanism of lightbased treatments is not known clearly, because the wavelengths, pulse widths, and energy vary depending on the type of light used. However, it is thought to involve the photodestruction of Cutibacterium acnes, damage to the sebaceous gland, or a peeling effect. ${ }^{1,4-6,8}$

For the treatment of acne vulgaris and enlarged facial pores, 1064-nm Q-switched Nd:YAG laser therapy assisted with topical application of a carbon suspension has been used. ${ }^{5-8}$ When a carbon suspension is applied on the face, some carbon particles penetrate the hair follicle and absorb the laser light, which directly heats the sebaceous glands to damage them. ${ }^{2}$ In addition, the laser energy and the peeling effect of carbon particles clean off the skin surface and clogged pores. ${ }^{2}$

Similarly, gold microparticles that are delivered to the follicle cause selective thermal damage to the infundibulum and sebaceous glands. Therefore, photothermal therapy using gold microparticles is commonly used for the treatment of acne vulgaris. ${ }^{1,2}$

In our cases, the microparticles we used were diamond particle suspensions and gold microparticles, which compensate for the disadvantages of carbon suspensions. Carbon suspensions cause a significant amount of odor and dust, because carbon particles break during laser procedures, causing discomfort to both medical staff and patients. However, in the case of diamond particle suspensions, the laser procedure can be more precise, because the particles are not easily broken.

Conventional carbon peeling induces fine peeling on the skin, brightens the skin texture, and removes sebum and dead cells from the facial pores. ${ }^{5,7,8}$ In addition, it is effective in improving inflammatory acne and removing comedones. ${ }^{5,6}$ We assumed that these reactions could also be elicited through the application of a diamond particle suspension. Interestingly, our laser therapy using diamond particle suspensions improved the patients' acne, decreased the excessive sebum secretion, improved the overall skin texture, and reduced the size of the enlarged pores. Furthermore, it visually improved the post-inflammatory hyperpigmentation and erythema on the face. 
Therefore, it can be hypothesized that diamond particle suspensions have a similar peeling effect with that of conventional carbon peeling. In particular, the microparticles used also contained gold, which may be delivered to facial pores and may have had a photothermal effect by absorbing the laser and damaging the sebaceous glands. ${ }^{1,2,9}$

In conclusion, the two reported cases demonstrate the potential of 1064-nm Q-switched Nd:YAG laser therapy assisted with diamond particle suspension and gold microparticle application as an effective treatment for acne vulgaris and enlarged facial pores; nevertheless, further studies are needed to compare this method with therapy using conventional carbon suspensions.

\section{CONFLICT OF INTEREST}

No potential conflict of interest relevant to this article was reported.

\section{FUNDING}

None.

\section{ORCID}

Hee Ung Park, https://orcid.org/0000-0001-9018-7208

Hangrae Cho, https://orcid.org/0000-0002-3913-1396

Sang Ju Lee, https://orcid.org/0000-0003-2228-7121

Han Kyoung Cho, https://orcid.org/0000-0001-6074-013X

\section{REFERENCES}

1. Paithankar DY, Sakamoto FH, Farinelli WA, Kositratna G, Blomgren RD, Meyer TJ, et al. Acne treatment based on selective photothermolysis of sebaceous follicles with topically delivered light-absorbing gold microparticles. J Invest Dermatol
2015;135:1727-34

2. Park KY, Han HS, Hong JY, Seo SJ, Lee SJ. Gold nanoshellmediated photothermal therapy for acne vulgaris. Dermatol Ther 2020;33:e13189.

3. Fox L, Csongradi C, Aucamp M, du Plessis J, Gerber M. Treatment modalities for acne. Molecules 2016;21:1063.

4. Lloyd JR, Mirkov M. Selective photothermolysis of the sebaceous glands for acne treatment. Lasers Surg Med 2002;31:115-20.

5. Jung JY, Hong JS, Ahn CH, Yoon JY, Kwon HH, Suh DH. Prospective randomized controlled clinical and histopathological study of acne vulgaris treated with dual mode of quasi-long pulse and Q-switched 1064-nm Nd:YAG laser assisted with a topically applied carbon suspension. J Am Acad Dermatol 2012;66:626-33.

6. Guida S, Fulgione E, D'Ambra I, Babino G, Pellacani G, Farnetani F. Carbon peel laser technique to improve skin quality: back to science! Dermatol Pract Concept 2020;10:e2020113.

7. Chung H, Goo B, Lee H, Roh M, Chung K. Enlarged pores treated with a combination of Q-switched and micropulsed $1064 \mathrm{~nm}$ $\mathrm{Nd}$ :YAG laser with and without topical carbon suspension: a simultaneous split-face trial. Laser Ther 2011;20:181-8.

8. Guida S, Longhitano S, Galadari H, Spadafora M, Urtis GG, Pellacani $G$, et al. Resurrection of a new old technique: the carbon peel laser. Dermatol Ther 2020;33:e13646.

9. Park KY, Han HS, Park JW, Lee HW, Seo SJ. Gold photothermal therapy for refractory papulopustular rosacea: a case series. Photodermatol Photoimmunol Photomed 2021;37:243-6.

How to cite this article: Park HU, Cho H, Lee SJ, Cho HK. Use of 1,064-nm Q-switched neodymium:yttriumaluminum-garnet laser therapy assisted with diamond particle suspension and gold microparticle application for acne vulgaris and enlarged facial pores. Med Lasers 2021; 10:242-245. https://doi.org/10.25289/ML.2021.10.4.242 